\title{
Using Web Services to Link Electronic Clinical Records to Pharmacovigilance Databases
}

\author{
Ines Ribeiro-Vaz ${ }^{1,2 *}$ and Ricardo J C Correia ${ }^{2,3}$ \\ ${ }^{* 1}$ Northern Pharmacovigilance Centre, Faculty of Medicine, University of Porto, Portugal (entity funded by Infarmed - National Authority of Medicines and \\ Health Products, IP) \\ ${ }^{2}$ Centre for Research in Health Technologies and Information Systems (CINTESIS) University of Porto, Portugal \\ ${ }^{3}$ Health Information and Decision Sciences Department (CIDES), Faculty of Medicine, University of Porto, Portugal
}

Received: August 08, 2016; Accepted: September 30, 2016; Published: October 05, 2016

*Corresponding author: Ines Ribeiro-Vaz, Northern Pharmacovigilance Centre, Faculty of Medicine, University of Porto, Portugal, E-mail: inesribeirovaz@gmail.com

\begin{abstract}
Purpose: Adverse Drug Reaction (ADR) reporting is an efficient method to assess the safety of drugs. However, underreporting is a major issue undermining the effectiveness this method.Among patients with Inflammatory Bowel Disease (IBD), ADR are a serious problem, because these patients are often treated with new and potent drugs. Electronic registries usually include information on ADR, recorded by physicians. To promote ADR reporting by gastroenterologists working in a multicentre IBD study group, we proposed the utilisation of a web service in their usual Electronic Health Records (EHR) to collect ADR. The aim of this work was to describe the impact of this intervention on the number of ADR reported to the regulatory authority through a regional pharmacovigilance centre.
\end{abstract}

Methods: A study was performed between 2013 and 2015. A web service was created and implemented in an EHR in use. We analysed the trends and type of ADR reported through this service.

Results: From April 2013 to February 2015, 167 ADR reports were sent to the Northern Pharmacovigilance Centre through the web service, comprising $10 \%$ of the total ADR reports received in the same period. Of these, 118 cases were serious (one was life-threatening). In the northern region of Portugal, GEDII physicians reported 9 ADR during the 23 months previous to web service implementation and 121 ADR during the 23 months after web service implementation, i.e. an increase of $1244 \%$.

Conclusions: This solution allowed for reporting 167 ADR during the first 23 months of implementation, simply by clicking a button included in the usual EHR used by gastroenterologists. These results suggest that information systems (IS) should facilitate the reporting of ADR.

Keywords: Adverse Drug Reactions; Inflammatory Bowel Disease; Pharmacovigilance; Electronic Health Records

\section{Introduction}

All medicines have adverse effects, some of them unknown until the drug is commercialised. Thus, it is crucial to implement strategies to monitor drug safety. Pharmacovigilance is the activity of drug surveillance, after its launch onto the market, with the main goal of public health protection, ensuring that the drug benefit outweighs its risks. Worldwide, pharmacovigilance systems are mostly based on spontaneous ADR reports made by healthcare professionals and consumers. Spontaneous ADR reporting has been described as an essential method to detect drug safety signals; however, underreporting is a major issue undermining the effectiveness of spontaneous reports. Several studies suggest that fewer than $10 \%$ of detected ADR are effectively reported to medicine regulatory authorities (e.g. Food and Drug Administration - FDA, European Medicines Agency EMA, etc.) [1,2].

It is known that, one of the main reasons why healthcare professionals do not report ADR is due to an increase in their workload $[3,4]$. So, in order to reduce ADR reporting efforts, Information Systems (IS) to promote ADR reporting or to detect ADR in healthcare institutions have been tested and used, such as software for voluntary and automated detection of ADR, tools that analyse clinical databases or web-sites that actively inform healthcare professionals [5]. Information and communication technologies can also be used to facilitate and promote ADR reporting, such as the creation of on-line reporting forms and the development of tools to collect safety data from Electronic Health Records (EHR), among others [6,7].

In Portugal, there is a multi centre research project, in the field of gastric diseases (Study Group of Inflammatory Bowel Disease GEDII) [8] whose members use the same electronic health record to collect patient information. As these patients are often treated with new and potent drugs (e.g. immunomodulating agents), the EHR has a field related to medication and ADR. Since the group members already fill in this field, it was considered an advantage to create a tool to send the data to the pharmacovigilance system. Aim

The aim of this work was to implement and describe the implementation of a web service in an EHR to collect ADR reports and analyse the number of ADR reports sent to the Portuguese regulatory authority (INFARMED), through a regional pharmacovigilance centre. 


\section{Methods}

\section{Intervention}

The multi-centre research project, the Study Group of Inflammatory Bowel Disease - GEDII [8], has its own Electronic Health Record (EHR) to collect patient information, with a field related to medications and ADR.

The GEDII members asked for collaboration with the Northern Pharmacovigilance Centre (UFN in Portuguese) and the Health Information and Decision Sciences Department (CIDES) of the Faculty of Medicine to develop a tool to allow for easy reporting of ADR included in the EHR to the pharmacovigilance system. The two entities had, at the time, released a new information service (a web service) to collect suspected adverse reactions directly from prescription and medical records. This system was easily adapted to the EHR used by the GEDII group.

The Northern Pharmacovigilance Centre, as part of the Portuguese Pharmacovigilance System, receives ADR reports from healthcare professionals (and, since 2012, from consumers as well) through an on-line form, a paper form, e-mail and by telephone. Since 2013, UFN has also received ADR reports through the web service.

The web service anonymises patient data (converting the full name of the patient into initials), according to the data protection standards of the Portuguese Pharmacovigilance System. Figure 1 shows the information flow from the electronic health record to the pharmacovigilance system.

To use this service, there is a button asking the doctor if he/ she will allow the information to be sent to the pharmacovigilance database (see Figure 2). If the physician does not allow for this information to be sent to the system, it will only be stored in the health record.

\section{Study design and data collection}

The web service was implemented in the GEDII electronic health record (used only by gastroenterologists) in April 2013. There are 15 hospitals using this information system, covering a total of 4031 patients. The database has 39 registered users, which means that, potentially, 39 physicians could report ADR through this information system [8].
In order to use this web service, it is necessary to access a specific URL, provided by the Northern Pharmacovigilance Centre and CIDES. The service was incorporated in the GEDII software to collect the ADR already stored by the physicians in the system. Each physician, in the context of the patient, sends the ADR to the Regional Pharmacovigilance Centre.

After the ADR report is received by the Regional Pharmacovigilance Centre, their technical staff process the report according to the pharmacovigilance guidelines [9], sending it to the Portuguese Regulatory Authority (INFARMED).

If the doctor does not want to send the information to the pharmacovigilance system, it will only be stored in the medical history.

To analyse the trends and type of ADR reported through this service, we performed a descriptive analysis on the number and seriousness of ADR reported. The seriousness was assessed using the World Health Organisation [10] seriousness criterion [11]. According to this criterion, a serious ADR is any untoward medical occurrence that at any dose: results in death, requires inpatient hospitalisation or prolongation of existing hospitalisation, results in persistent or significant disability/ incapacity or is life-threatening.

\section{Results}

From April 2013 to February 2015, physicians from GEDII reported 167 ADR through the described web service.

Of the 167 reported ADR, 118 (71\%) were serious ADR, considering the World Health Organisation seriousness criterion [11]. One of the cases was life-threatening and none were fatal.

To calculate the increase in ADR reporting in this period, we used data from the northern region, which is the delimited area of the Northern Pharmacovigilance Centre, as we did not have access to national ADR reporting data. Thus, considering the physicians from the GEDII group that work in at the northern region of Portugal, 9 ADR were reported during the 23 months prior to web service implementation and 121 ADR were reported during the 23 months after web service implementation, i.e. an increase of $1244 \%$.

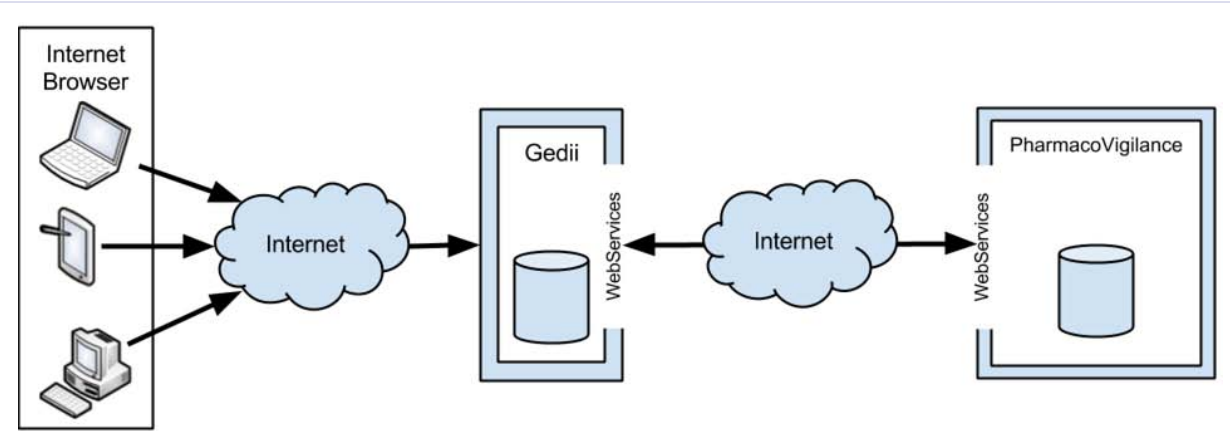

Figure 1: Information flow 


\section{Drugs involved in the Reported ADR}

Most of the cases had one only suspected drug, but in six cases there were two suspected drugs involved.

Of the reported ADR, 106 cases (63\%) were presumably due to azathioprine, 23 cases (14\%) to infliximab, 14 cases (8\%) to adalimumab and $9(5 \%)$ cases to methotrexate (Figure 3$)$.

All of these drugs are classified as antineoplastic and immunomodulating agents, according to the WHO Anatomical Therapeutic Chemical (ATC) classification system [12]. Azathioprine, infliximab and adalimumab are immunosuppressants and methotrexate is an antimetabolite.

\section{Discussion}

Worldwide, many systems use electronic health records to facilitate adverse event reporting, with the same goal as our work, which is to not overload healthcare professionals with additional administrative work to report ADR ${ }^{[7,13,14]}$. These systems can be included in the EHR and be easily completed [13] or can actively help in form filling with the automatic input of certain information already included in the EHR [7]. During the ALIAS (Albumin in Acute Stroke Trial) experience, authors decided to integrate an electronic safety reporting module into the existing web-based system to deal with safety reporting obligations of a multicentre clinical trial [15]. This system pre-populates the reporting form with the existing information, which needs to be completed and validated by clinical staff. The ASTER pilot study also brought an important contribution to the development of these systems, as it triggered ADR within the EHR, collected patient data, populated the ADR reporting form and made the report available to the physician for review [16]. This work is different from ours, because it asks the clinician to provide additional information on the adverse event and then submits the report. All these systems incur extra work on the part of physicians, which is difficult to eliminate. The novelty of our work is the detail of allowing the physician to report ADR without any additional administrative work beyond the usual clinical registry. In addition, the EHR remained the same, without the need to change the physicians' routine. This system adapts to the routines of the healthcare professionals and not vice versa, which can explain the increasing of ADR reporting among the studied group.

It is also important to note that most of the reported ADR were serious. In fact, the studied group deals with innovative and powerful drugs those often cause serious adverse events. This finding reinforces the importance of our work, as Pharmacovigilance Systems seek mainly information about serious (and unexpected) ADR [17].

Our study has some limitations, such that it only included gastroenterologists. However, this web service is available to be used in several software. Even so, it is currently in use only in two software systems: the one described in this study and an electronic prescription software system. Another issue is that our study does not have a control group, which limits the conclusions drawn. For this reason, the only comparison that the authors were able to perform was for the number of ADR reports made by the same group of gastroenterologists before the web service implementation.

\section{Conclusions}

The simple solution described in our work allowed for

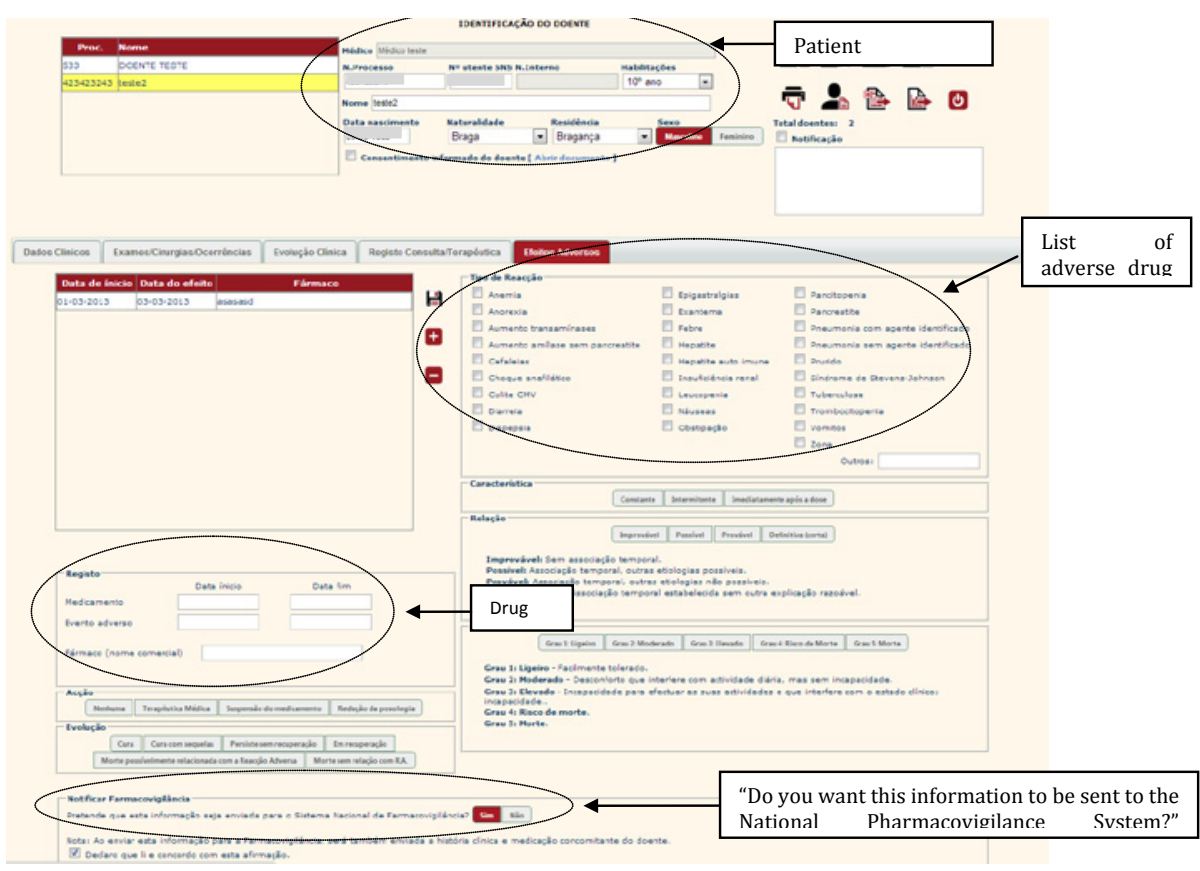

Figure 2: Screen shot of the ADR section on the GEDII electronic health record 


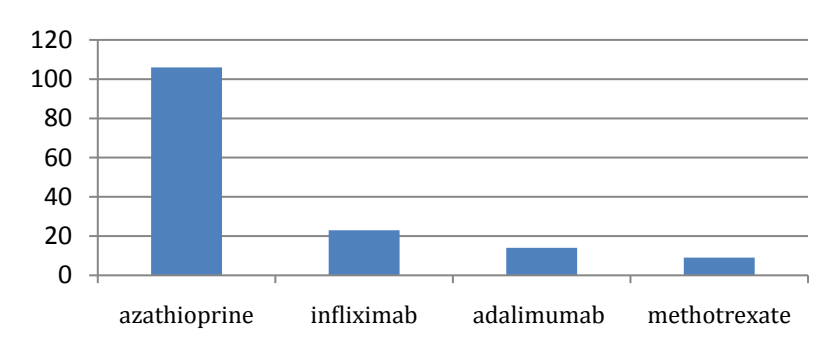

Figure 3: Drugs involved in the reported ADR

reporting 167 ADR during the first 23 months of implementation, simply by clicking a button included in the usual electronic health record used by gastroenterologists. Our results suggest that doctors would report more ADR if they do not have to take on any further workload to do it. We propose that IS used to support multicentre studies should be used to report detected ADR.

\section{Author Contributions}

Inês Ribeiro-Vaz was responsible for data collection and writing the paper. Ricardo J C Correia conceptualised the intervention and supervised writing of the paper.

\section{Conflict of Interest}

The authors declare that they have no conflicts of interest.

\section{References}

1. McGettigan P, Golden J, Conroy RM, Arthur N, Feely J. Reporting of adverse drug reactions by hospital doctors and the response to intervention. Br J Clin Pharmacol. 1997;44(1):98-100.

2. Hazell L, Shakir SA. Under-reporting of adverse drug reactions : a systematic review. Drug Saf. 2006;29(5):385-96.

3. Hinrichsen VL, Kruskal B, O’Brien MA, Lieu TA, Platt R. Using electronic medical records to enhance detection and reporting of vaccine adverse events J Am Med Inform Assoc. 2007;14(6):731-5.

4. Lee SB, Schepers GP, Goldberg KL. Electronic adverse-drug-reactionreporting program. Am J Health Syst Pharm. 2004;61(12):1230, 12323.

5. Molokhia M, Tanna S, Bell D. Improving reporting of adverse drug reactions: Systematic review. Clin Epidemiol. 2009;1:75-92.
6. Ribeiro-Vaz I, Santos C, Da Costa-Pereira A, Cruz-Correia R. Promoting spontaneous adverse drug reaction reporting in hospitals using a hyperlink to the online reporting form: An ecological study in Portugal. Drug Saf. 2012;35(5):387-94. doi: 10.2165/11597190-00000000000000 .

7. Ortega A, Aguinagalde A, Lacasa C, Aquerreta I, Fernandez-Benitez $M$, Fernandez LM. Efficacy of an adverse drug reaction electronic reporting system integrated into a hospital information system. Ann Pharmacother. 2008;42(10):1491-6. doi: 10.1345/aph.1L130.

8. Study Group of Inflammatory Bowel Disease. Grupo de Estudos da Doença Inflamatória Intestinal (GEDII) 2014 [cited 2014]. Available from: www.gedii.pt.

9. European Medicines Agency. European Medicines Agency 2014 [cited 2014 05-09-2014]. Available from: www.ema.europa.eu.

10. McLawhorn MW, Goulding MR, Gill RK, Michele TM. Analysis of Benzonatate Overdoses Among Adults and Children from 1969-2010 by the United States Food and Drug Administration. Pharmacotherapy. 2013;33(1):38-43. doi: 10.1002/phar.1153.

11.World Health Organization. The Uppsala Monitoring Centre Uppsala WHO Collaborating Centre for International Drug Monitoring [17 July 2015]. Available from: http://www.who-umc.org/DynPage. aspx?id=22682.

12.World Health Organization. WHO Collaborating Centre for Drug Statistics Methodology 2014 [cited 2015 03-2015]. Available from: http://www.whocc.no/.

13. Haw C, Cahill C. A computerized system for reporting medication events in psychiatry: the first two years of operation. J Psychiatr Ment Health Nurs. 2011;18(4):308-15. doi: 10.1111/j.1365-2850.2010.01664.x.

14. Hickner J, Zafar A, Kuo GM, Fagnan LJ, Forjuoh SN, Knox LM, et al. Field test results of a new ambulatory care Medication Error and Adverse Drug Event Reporting System-MEADERS. Ann Fam Med. 2010;8(6):517-25. doi: 10.1370/afm.1169.

15. Zhao W, Waldman BD, Dillon C, Pauls K, Kim J, Patterson L, et al. A webbased medical safety reporting system for a large multicenter clinical trial: The ALIAS experience. Contemp Clin Trials. 2010;31(6):536-43. doi: 10.1016/j.cct.2010.08.010.

16. Linder JA, Haas JS, Iyer A, Labuzetta MA, Ibara M, Celeste $M$, et al. Secondary use of electronic health record data: spontaneous triggered adverse drug event reporting. Pharmacoepidemiol Drug Saf. 2010;19(12):1211-5.

17. Pharmacovigilance in Portugal. Lisbon: INFARMED - National Authority of Medicines and Health Products. I.P; 2004;526. 